Received: $\quad 2015.07 .24$ Accepted: 2015.09.23 Published: 2015.10 .23

Authors' Contribution: Study Design A Data Collection B Statistical Analysis C Data Interpretation D Manuscript Preparation E Literature Search F Funds Collection G
Corresponding Author: Source of support:

\section{Beneficial Effect of Probiotics Administration in Inflammatory Bowel Disease and Related Spondyloarthropathy: A Prospective Study}

\author{
ADE 1,2 Giovanni Tomasello \\ AE 3 Giuseppe Margiotta \\ DF 4 Marcello Noto \\ $C D$ 2,5 Emanuele Sinagra \\ AB 6 Maurizio Filippo Accardo \\ ABF 7 Provvidenza Damiani \\ CD 4 Vincenza Maria Arculeo \\ ADF 8 Francesca Guarneri \\ AB 3 Antonino Sanfilippo \\ AG 1,2 Francesco Cappello \\ ADE 1,9 Giovanni Grasso
}

1 Department of Experimental Biomedicine and Clinical Neurosciences, University of Palermo, School of Medicine, Palermo, Italy

2 Euro-Mediterranean Institute of Science and Technology (IEMEST), Palermo, Italy 3 Orthopedic and Traumatology Unit, University of Palermo, Palermo, Italy 4 AOUP Azienda Universitaria Policlinico, Palermo, Italy

5 Gastroenterology and Endoscopy Unit, Fondazione Istituto San Raffaele, G. Giglio, Cefalù, Italy

6 KORE University, Enna, Italy

7 Geriatric Unit, Department DIBIMIS, University of Palermo, Palermo, Italy

8 Department of Obstetrics and Gynecology, University of Palermo, Palermo, Italy 9 Neurosurgical Unit, University of Palermo, Palermo, Italy

Background: Therapeutic treatment for inflammatory bowel disease (IBD) may follow different approaches, systemic as well as local, especially when the target is the osteoarticular apparatus. Although some clinical trials have suggested benefits from probiotics administration in IBD, there is still a great deal of controversy on their use. The aim of this study was to further investigate the effects of probiotics in patients with IBD and related spondyloarthropathy.

Material/Methods: Fifty-nine patients affected by IBD with extra-intestinal involvement where evaluated from 2006 to 2010. Twenty-eight patients received administration of a standard therapy with mesalazine and 31 where treated with the same therapy plus probiotic supplement.

Results: Comparative analysis performed between the 2 groups showed a better clinical response and a reduction of occasional corticosteroids administration in patients receiving probiotic administration.

Conclusions: This prospective study supports the beneficial effects of probiotic administration in improving the clinical response to standard therapy and in reducing the need for corticosteroids use. Large randomized trials would provide reliable and conclusive results in this field.

MeSH Keywords:

Arthritis • Inflammatory Bowel Diseases • Microbiota • Probiotics

Full-text PDF: http://medscitechnol.com/abstract/index/idArt/895438

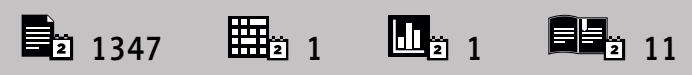




\section{Background}

The ulcerative colitis (UC) and the Crohn's disease (CD) are the principal pathological entities of inflammatory bowel disease (IBD). They are characterized by an aberrant inflammatory reaction towards the microbiota in the gastro-intestinal tract [1]. The pathogenetic mechanisms underlying the inflammatory response are still under investigation. The role of exogenous factors (e.g., microbiota, infective agents, lifestyle, and eating) and endogenous factors (e.g., genetic predisposition, and barrier function of the mucous membrane) have been discussed [2]. In UC, a large inflammatory infiltrate, mainly represented by neutrophils, is observed both in the mucous membrane and the under-located membrane [3]. Most secreted cytokines are interleukin-4, interleukin-5, and interleukin-13. Crypts and serpiginous painful blisters spreading throughout the rectum, with the growth of pseudo polyps, are often encountered. The latter are caused by the continuous regeneration of cells into the basal layer [3]. In CD, all the layers of the intestinal wall are involved, with a segmental distribution of the painful blisters, distinct from the inflammatory granulation tissue generated by the action of gamma interferon and TNFalpha. In this scenario stenosis, fistulas with the neighboring organs (e.g., pancreas and liver), and abscesses are seen [3].

Patients affected by IBD may present extra-intestinal manifestation (e.g., articular involvement), with a reported prevalence between $17 \%$ and $39 \%$ [3]. In this regard, involvement of the axial joints and peripheral arthritis are frequently reported and therapeutic management is mainly addressed at reducing the inflammation and preventing disability. This requires a multimodal treatment based on the combination of exercise and non-steroidal anti-inflammatory drugs. Although immunomodulators have been reported to be efficacious in patients with peripheral arthritis and other extra-intestinal manifestations, no beneficial effects have been found for the treatment of axial symptoms of spondylitis [3].

Here, we report the result of a clinical trial investigating the effects of probiotic use on clinical outcome in patients with IBD.

\section{Material and Methods}

Fifty-nine patients affected by IBD with extra-intestinal involvement where enrolled from 2006 to 2010 . Twenty-eight patients (Group $A$ ) received administration of a standard therapy with mesalazine and nonsteroidal anti-inflammatory agents and 31 patients (Group B) where treated with the same therapy plus probiotic administration. Both groups received mesalazine in gastro-resistant tablets in a dosage of $800 \mathrm{mg}$ twice a day for mild forms or 3 times a day for moderate forms. Patients affected by IBD-related spondyloarthropathy were given diclofenac in a dose of $75 \mathrm{mg}$ a day for 10 days. Group B patients received probiotics plus standard therapy, in a standardized approach with intervals of 2 weeks for a total of 52 weeks. During the first week of treatment, patients were given a tablet in the morning and one in the evening, containing a mixture of Enterococcus faecium and Saccharomyces boulardii to mitigate the intestinal inflammation. In the second week they received a tablet in the morning and one in the evening, containing a mixture of Lactobacillus salivarius and Lactobacillus acidophilus to ensure a normal intestinal microenvironment. Both groups received additional corticosteroid treatment (tablets of $25 \mathrm{mg}$ ) when necessary.

The study was approved by the local ethics committee. All patients were clearly informed and signed a written informed consent form. The patients were not asked to stop their other medications during the study.

Comparative analysis between the 2 groups was performed assessing the Crohn's Disease Activity Index (CDAl) [4] and the Western Ontario and McMaster Universities Arthritis (WOMAC) index [5]. The chi-square test was used to demonstrate a correlation between the reduced use of cortisone and the probiotic therapy,. Unless otherwise indicated, all results are displayed as means \pm SEM of replicates. $P<0.05$ was considered significant.

\section{Results}

Fifty-nine patients (mean age 43.4 years, range $17-68$ years) affected by IBD with extra-intestinal involvement where analyzed from 2006 to 2010 .

In this prospective study, patients were divided into 2 groups according to a random procedure and were evaluated with CDAI and WOMAC scales before beginning any therapy. The total average score measured in each patient was compared with the values obtained in the subsequent follow-up. By using the asymmetry calculation and arguing that the measured values were distributed according to the Gaussian curve, we used the $t$ test to demonstrate whether the clinical difference in improvement in Group B and Group A was statistically significant.

In the entire period of observation, the average score obtained by the CDAl score was 348.75 for Group A and 242.5 for Group $B(P<0.05)$ (Figure $1 A)$. The WOMAC score was also significantly different between the groups, being 73.5 in Group $A$ and 53.38 in Group B $(P<0.05)$ (Figure $1 B)$. Patients in Group B received less cortisone administration than patients in Group $A$ $(P<0.05)$. Data are shown in Table 1. 


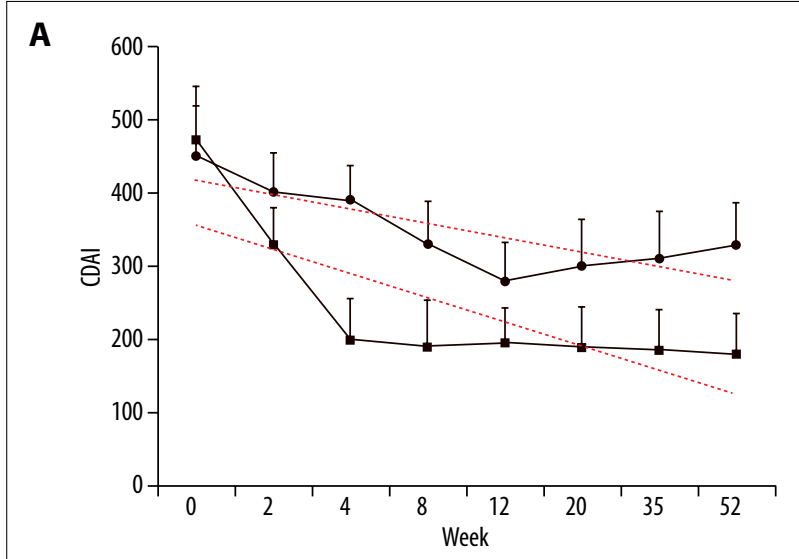

B

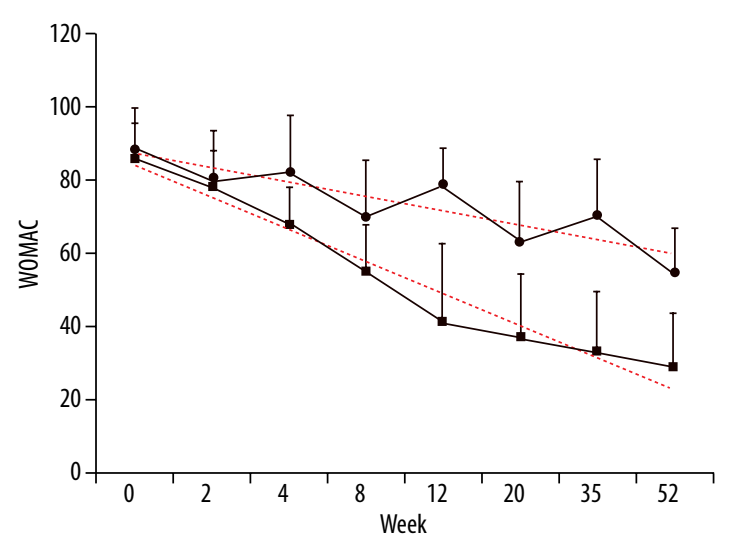

Figure 1. Line graphs showing the average CDAI (A) and WOMAC (B) score in the entire period of observation for both treatment groups. Overall, the mean CDAI was 348.75 for Group $A$ and 242.5 for Group $B(P<0.05)$. The WOMAC score was also significantly different between the groups, being 73.5 in Group $A$ and 53.38 in Group $B(P<0.05)$.

Table 1. Cortisone administration between the groups.

\begin{tabular}{ccccc} 
& Cortisone & No cortisone & Total \\
\hline Group A & 19 & 9 & 28 \\
\hline Group B & 7 & 24 & 31 \\
\hline Total & 26 & 33 & 59 \\
\hline
\end{tabular}

The correlation between the reduced use of cortisone and the probiotic therapy was assessed by the Chi-square test (Chi squared=10.468; $P<0.05)$.

\section{Discussion}

Inflammatory bowel disease (IBD) is a chronic inflammatory pathology involving the gastrointestinal tract and comprising ulcerative colitis (UC) and Crohn's disease (CD). IBD is diagnosed in 2.5-3 million people in Europe and more than 1 million people in the United States $[4,5]$. Therapeutic management traditionally involves the use of anti-inflammatory agents (e.g., mesalazine, corticosteroids, and immunomodulators) and biologic agents. The exact pathophysiological mechanisms underlying IBD are unknown. However, the role of an abnormal intestinal immune response to the gut microbiota in a genetically susceptible host has been reported [6]. Accordingly, the interest in microbiota-based therapy has blossomed in recent years, due in part to having fewer adverse effects than traditional therapy. The microbiota is an ensemble of microorganisms that resides in the gastro-intestinal tract to form a "tissue into the organ". It establishes a relation of mutual benefit with the gastro-intestinal tract through its metabolic activities. The microbiota exerts various functions, such as the inhibition of pathogenic bacteria and the synthesis of short-chain fatty acids. It promotes the absorption of nutrients and minerals, acts as an immune system to the mucous membrane, and supports the synthesis of vitamins and amino acids and the metabolism of proteins.
Most of the bacterial population forming the microbiota is not pathogenic. However, the balance between pathogenic and non-pathogenic bacteria promotes a positive influence in favor of the gastro-intestinal tract. Alteration of this equilibrium can produce dysbiosis [7] and IBD. In particular, the change of a bacterial microflora into a saprophytic bacteria population may alter tight junctions, which are responsible for the correct functioning of the epithelial surface in the intestinal mucosa. Accordingly, the antigenic intercellular passage from the lumen in the mucosa activates an immune system response allowing the inflammatory cascade and production of cytokines to be initiated. Given this close relationship between the intestinal microflora and IBD, several studies have investigated the use of probiotics, prebiotics, and synbiotics to positively influence the microbiota to change or improve the classical IBD therapy.

In this prospective study, patients receiving standard therapy plus probiotics administration presented with a significantly better outcome and reduction in occasional corticosteroids use than those treated by standard therapy. Our findings are in agreement with several studies suggesting that the use of probiotic products has beneficial effects on gut function and microbiota in patients with IBD [8-10]. However, some conflicting data have been also reported [11]. 
With the introduction of modern technologies, the understanding of the etiopathogenesis of IBD has radically changed over the years. An increasing number of studies have shown that the starting point of the inflammatory process in IBD is dysbiosis of the gut microbiome, which may be produced by host genetics and environmental factors like diet. The next step is the understanding of the mechanisms of the host immune defenses that determine this intriguing host-microbiome ecology. In particular, a clear knowledge of the relationship between the genotype of the host and the extent to which it determines the microbiome characteristics will provide further insights. It will help in tailoring custom therapeutic interventions, based on the knowledge of the host genotype and the microbiome composition to choose the best strategy for treating patients affected by IBD.

\section{Conclusions}

This prospective study shows the beneficial effects of probiotic administration in improving the clinical response to standard therapy and in reducing the need for corticosteroids use in patients affected by IBD. Large randomized trials would provide reliable and conclusive results in this field.

\section{References:}

1. Palm O, Moum B, Jahnsen J et al: The prevalence and incidence of peripheral arthritis in patients with inflammatory bowel disease, a prospective population-based study (the IBSEN study). Rheumatology, 2001; 40: 1256-61

2. Abraham C, Cho JH: Inflammatory bowel disease. New Engl J Med, 2009; 361: 2066-78

3. Mendoza JL, Lana R, Taxonera C et al: [Extraintestinal manifestations in inflammatory bowel disease: differences between Crohn's disease and ulcerative colitis]. Med Clin (Barc), 2005; 125: 297-300 [in Spanish]

4. Kappelman MD, Rifas-Shiman SL, Kleinman K et al: The prevalence and geographic distribution of Crohn's disease and ulcerative colitis in the United States. Clin Gastroenterol Hepatol, 2007; 5: 1424-29

5. Orel R, Kamhi T, Vidmar G et al. Epidemiology of pediatric chronic inflammatory bowel disease in central and western Slovenia, 1994-2005. J Pediatric Gastroenterol Nutr, 2009; 48: 579-86

6. Tomasello G, Bellavia M, Palumbo VD et al: From gut microflora imbalance to mycobacteria infection: is there a relationship with chronic intestinal inflammatory diseases? Ann Ital Chir, 2011; 82: 361-68

7. Hawrelak JA, Myers SP: The causes of intestinal dysbiosis: a review. Altern Med Rev, 2004; 9: 180-97

8. Cui HH, Chen CL, Wang JD et al: Effects of probiotic on intestinal mucosa of patients with ulcerative colitis. World J Gastroenterol, 2004; 10: 1521-25

9. Venturi A, Gionchetti P, Rizzello F et al: Impact on the composition of the faecal flora by a new probiotic preparation: preliminary data on maintenance treatment of patients with ulcerative colitis. Aliment Pharmacol Ther, 1999; 13: 1103-8

10. Shadnoush M, Hosseini RS, Khalilnezhad A et al: Effects of probiotics on gut microbiota in patients with inflammatory bowel disease: A doubleblind, placebo-controlled clinical trial. Korean J Gastroenterol, 2015; 65(4): 215-21

11. Saez-Lara MJ, Gomez-Llorente C, Plaza-Diaz J et al: The role of probiotic lactic acid bacteria and bifidobacteria in the prevention and treatment of inflammatory bowel disease and other related diseases: a systematic review of randomized human clinical trials. BioMed Res Int, 2015; 2015: 505878 